\title{
Spectroscopy of horizontal branch stars in NGC 6752
}

\section{Anomalous results on atmospheric parameters and masses $\star$}

\author{
C. Moni Bidin ${ }^{1,2}$, S. Moehler ${ }^{3}$, G. Piotto ${ }^{1}$, Y. Momany $^{4}$, and A. Recio-Blanco ${ }^{5}$ \\ 1 Dipartimento di Astronomia, Università di Padova, Vicolo dell'osservatorio 3, 35122 Padova, Italy \\ 2 Departamento de Astronomía, Universidad de Chile, Casilla 36-D, Santiago, Chile \\ e-mail: mbidin@das.uchile.cl \\ 3 European Southern Observatory, Karl-Schwarzschild-Str. 2, 85748 Garching, Germany \\ 4 INAF - Osservatorio Astronomico di Padova, Vicolo dell'osservatorio 2, 35122 Padova, Italy \\ 5 Observatoire de la Côte d'Azur, Dpt. Cassiopée, CNRS UMR 6202, BP 4229, 06304 Nice Cedex 04, France \\ Received 28 June 2007 / Accepted 8 August 2007
}

\section{ABSTRACT}

\begin{abstract}
Context. The determination of fundamental parameters for horizontal branch stars in Galactic globular clusters frequently gives puzzling results, pointing to the lack of our understanding of their atmospheric structure and the inadequate approximations by the models.

Aims. We wanted to measure effective temperatures, surface gravities, helium abundances, and masses for hot horizontal branch stars in NGC 6752 in order to compare the results with evolutionary predictions.

Methods. We used the ESO VLT-FORS2 facility to collect low-resolution spectra of 51 targets distributed along the horizontal branch. We determined atmospheric parameters, by comparison with theoretical models through standard fitting routines, and masses by basic equations.

Results. Results generally agree with previous works, although not always with the theoretical expectations for cooler stars $\left(T_{\text {eff }} \leq\right.$ $15000 \mathrm{~K}$ ). The calculated color excess is systematically lower than the literature values, pointing towards a possible underestimation of effective temperatures. Moreover, we find two groups of stars at $T_{\text {eff }} \sim 14000 \mathrm{~K}$ and at $T_{\text {eff }} \sim 27000 \mathrm{~K}$ that present anomalies with respect to the general trend and expectations. We suppose that the three peculiar bright stars at $T_{\text {eff }} \sim 14000 \mathrm{~K}$ are probably affected by an enhanced stellar wind. For the eight extreme horizontal branch stars at $T_{\text {eff }} \sim 27000 \mathrm{~K}$ that show unusually high masses, we find no plausible explanation.

Conclusions. While most of our results agree well with the predictions of standard horizontal branch evolution, we still have problems with the low masses we derive in certain temperature ranges. We believe that Kurucz ATLAS9 LTE model atmospheres with solarscaled abundances are probably inadequate for these temperature ranges. Concerning the group of anomalous stars at $T_{\text {eff }} \sim 27000 \mathrm{~K}$, a Kolmogorov-Smirnov test indicates that there is only an $8.4 \%$ probability that these stars are randomly drawn from the general distribution in the color-magnitude diagram. This is not conclusive but indicates that these stars could be both (and independently) spectroscopically and photometrically peculiar with respect to the general extreme horizontal branch population.
\end{abstract}

Key words. stars: horizontal-branch - stars: fundamental parameters - globular clusters: individual: NGC 6752

\section{Introduction}

Many decades have passed since when horizontal branch (HB) stars were identified as evolved stars of low initial mass (approximately $0.7-2 M_{\odot}$ ) presently burning helium in their core (Hoyle \& Schwarzschild 1955; Faulkner 1966). Still, many aspects of the evolution and internal structure of these stars are not fully understood. In particular, in the color-magnitude diagrams of Galactic globular clusters, many well-established observed features of HB stars are good example of this, as seen in the case of the different blue extensions from one cluster to another, which are partially dependent on metallicity (Sandage \& Wallerstein 1960) but not fully explained by it (Sandage \& Wildey 1967; van den Bergh 1967). In addition, the HB may show "jumps", where stars appear brighter than the theoretical expectations (Grundahl et al. 1999; Momany et al. 2002, 2004), or gaps, i.e. underpopulated regions along the HB (Sosin et al. 1997; Catelan et al. 1998; Ferraro et al. 1998; Piotto et al. 1999). Spectroscopy of HB stars opened new questions, such as the

* Based on observations with the ESO Very Large Telescope at Paranal Observatory, Chile (proposal ID 69.D-0682). unexplained presence of fast rotators among stars redder than the Grundahl et al. (1999) jump (Peterson 1983, 1985a,b; Behr 2003; Behr et al. 2000a,b; Recio-Blanco et al. 2004) and the absence of close binaries among hot extreme horizontal branch (EHB) stars (Moni Bidin et al. 2006a,b).

Traditionally, low-resolution spectroscopy of HB stars has focused on determining of their fundamental quantities such as atmospheric parameters. This is usually achieved by comparing observed spectra with grids of theoretical ones obtained from model atmospheres. As a consequence, any aspect of stellar structure unaccounted for in the model may be directly reflected on unexpected values measured for the parameters. For example, it is well known that surface gravities are systematically lower than expected in the temperature range $12000-20000 \mathrm{~K}$ if model spectra with the metallicity of the parent globular cluster are used. As a consequence, masses are derived that are too low (Moehler et al. 1995). This "mass discrepancy" can be partially explained by radiative levitation of heavy elements (Grundahl et al. 1999), originally proposed as the physical process resposible for abundance anomalies observed in field Ap stars (Michaud 1970). Precise calculations confirmed that this phenomenon 
should be at work in the atmospheres of these stars and can account for observed anomalies (Michaud et al. 1983), but the problem of the mass discrepancy is still not completely solved (Moehler 2001). On the other hand, Vink \& Cassisi (2002) point out that neglecting the presence of stellar wind can cause measured surface gravities to be erroneously low. Nevertheless, the derived values of stellar parameters - even if erroneous or unexpected - have been useful for pointing out where canonical models are inadequate, stimulating further investigations to solve the open problems.

As part of a program aimed at searching for close binaries among HB stars (Moni Bidin et al. 2006b, hereafter Paper I), we also collected low-resolution spectra of our target stars. In this paper we present the analysis of these spectra and critically discuss the derived atmospheric parameters (effective temperature, surface gravity, helium abundance) and stellar masses.

\section{Observations and data reduction}

The spectra were collected during two nights of observations (June 12 and 13, 2002) at the VLT-UT4 telescope equipped with the spectrograph FORS2 in MXU mode. The fifty-one target stars are the same objects already described in Paper I. They are distributed along the entire $\mathrm{HB}$, spanning a wide range in temperature from $T_{\text {eff }} \approx 8000 \mathrm{~K}$ up to $T_{\text {eff }} \approx 30000 \mathrm{~K}$. Coordinates and photometric data of the observed stars are presented in Table 1 of Paper I. Figure 1 shows the position of target stars in the color-magnitude diagram.

Two 1350s exposures were secured for each target with grism $600 \mathrm{~B}+22$ and 0.5 - wide slits for a resulting resolution of $3 \AA$. The spectral range was approximately $2900 \AA$ wide and, on average, centered at $4600 \AA$, but slightly different from star to star due to the different positions of the slits in the mask used for multi-object spectroscopy. All Balmer lines from $\mathrm{H}_{\beta}$ to $\mathrm{H} 10$ were always present in the spectra. We never used wavelengths shorter than $3600 \AA$ in our analysis, because of the lack of atmospheric transmission and instrumental response. In Fig. 2 we show an example of the reduced spectra.

The calibration images (bias, flat, and lamp) were acquired before and at the end of each observing night. Data reduction was performed with standard MIDAS ${ }^{1}$ procedures. All of the 2D spectra were trimmed from the frames with their calibration images and reduced independently. The wavelength calibration was performed with the $\mathrm{HeHgCd}$ lamp images, fitting a 3rd order polynomial to the dispersion relation. The rms error indicated by the fitting procedure was $0.07 \AA$ on average. Before extraction, we rebinned the $2 \mathrm{D}$ images to constant wavelength steps of $0.4 \AA /$ pix and corrected their curvature along the spatial axis by tracing the spectra as described in Moehler \& Sweigart (2006). Spectra were then extracted both with an optimum extraction algorithm (Horne 1986) and with a simple sum, and then, for each star, we chose the procedure that gave better results in terms of $\mathrm{S} / \mathrm{N}$, as the optimum algorithm sometimes failed to correctly extract the spectra of bright stars. We corrected them for atmospheric extinction, with the extintion coefficients for the La Silla observatory (Tüg 1977). The response curve was obtained with observations of the standard stars EG274 (both nights) and LTT3218 (second night only), using the flux tables of Hamuy et al. (1994). We had to use a high-order polynomial

${ }^{1}$ ESO-MIDAS is the acronym for the European Southern Observatory Munich Image Data Analysis System which is developed and maintained by the European Southern Observatory (http://www.eso.org/projects/esomidas/).

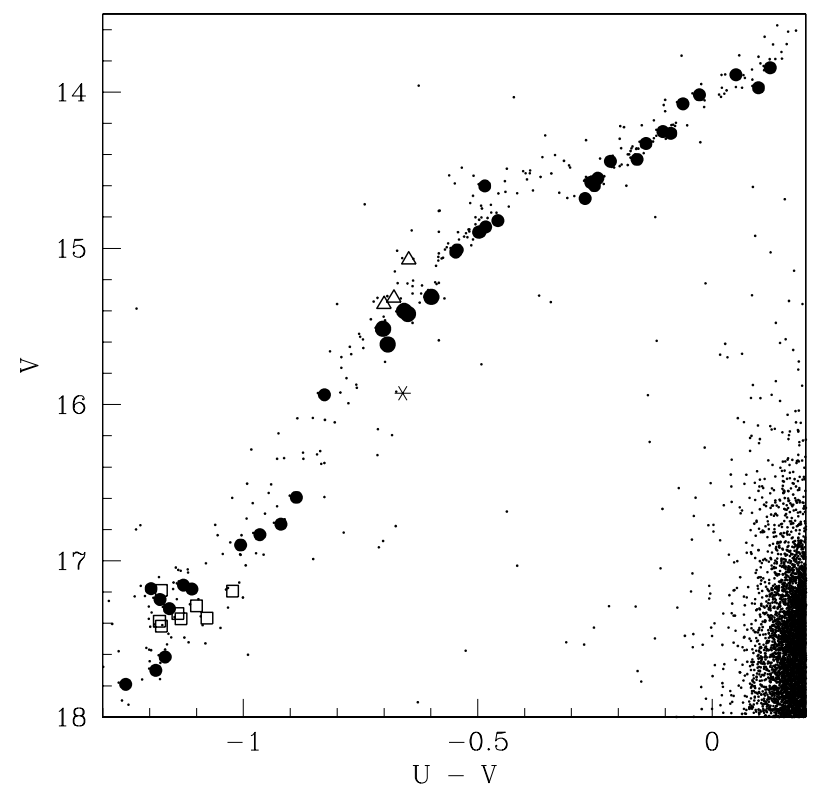

Fig. 1. Observed stars in the $V$ vs. $U-V$ color-magnitude diagram. Photometric data are from Momany et al. (2002). The stars are indicated with different symbols, as discussed in the text. The same symbols will be used in all of the figures to distinguish groups of stars with different properties.

(6th) so as to obtain a good response curve and a smooth continuum on the calibrated spectra. The resulting curves had the same shape with no difference between the nights. To calibrate the spectra of the second night we averaged the two corresponding curves when two standard stars were observed.

Finally, we corrected the spectra for radial velocity shifts. Absolute radial velocities of the targets were already measured on higher resolution spectra as described in Paper I, but we measured them again on each of the low-resolution spectra used in this work. In fact, as already discussed in Paper I, the shift of the spectra with respect to laboratory wavelength was due not only to the radial velocities of the stars, but also to many effects such as the position of the stars in the slitlets and systematics in wavelength calibration. We fitted the cores of all Balmer lines from $\mathrm{H}_{\beta}$ to $\mathrm{H}_{9}$ with a Gaussian profile, excluding $\mathrm{H}_{\epsilon}$ due to blending with the $\mathrm{Ca}$ II $\mathrm{H}$ line, and assumed the average as the radial velocity to be corrected.

Usually the values from single lines agreed within $10 \mathrm{~km} \mathrm{~s}^{-1}$, but in some cases there were large differences (up to $50 \mathrm{~km} \mathrm{~s}^{-1}$ ), an effect already described in Moehler et al. (1997). We checked if these could be ascribed at least in part to a rigid shift of the spectra on the CCD, constant in pixel and then variable with wavelength in $\mathrm{km} \mathrm{s}^{-1}$, but we found no evidence of a clear trend with wavelength.

\section{Measurements}

In order to derive effective temperatures, surface gravities, and helium abundances, we fitted the observed Balmer and Helium lines with stellar model atmospheres.

As discussed extensively in recent years, HB stars hotter than about 11000-12000 K show evidence of diffusion, e.g. deficiency of helium and strong (solar to super-solar levels) enrichment in iron (Grundahl et al. 1999; Glaspey et al. 1985, NGC 6397; Glaspey et al. 1989; Moehler et al. 2000, NGC 6752; Behr et al. 1999; Moehler et al. 2003, M 13; Behr et al. 2000a, M 15; Fabbian et al. 2005, NGC 1904; 


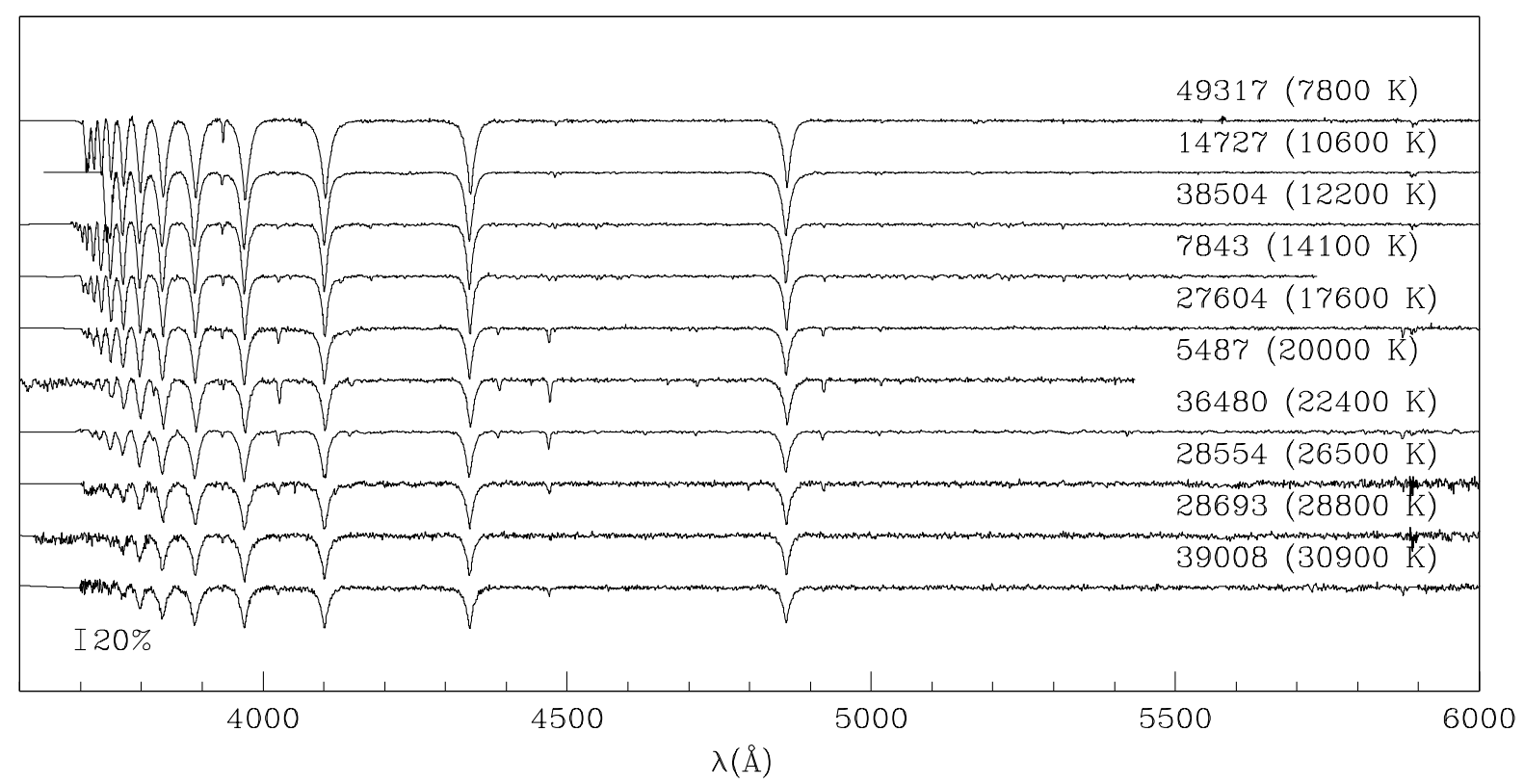

Fig. 2. A sample of the collected spectra, normalized and ordered by increasing $T_{\text {eff }}$ from top to bottom. Spectra were normalized fitting a 3rdorder spline function to the continuum. For wavelengths shorter than $3900 \AA$, where Balmer lines blend, the fit was anchored at the highest points between lines.

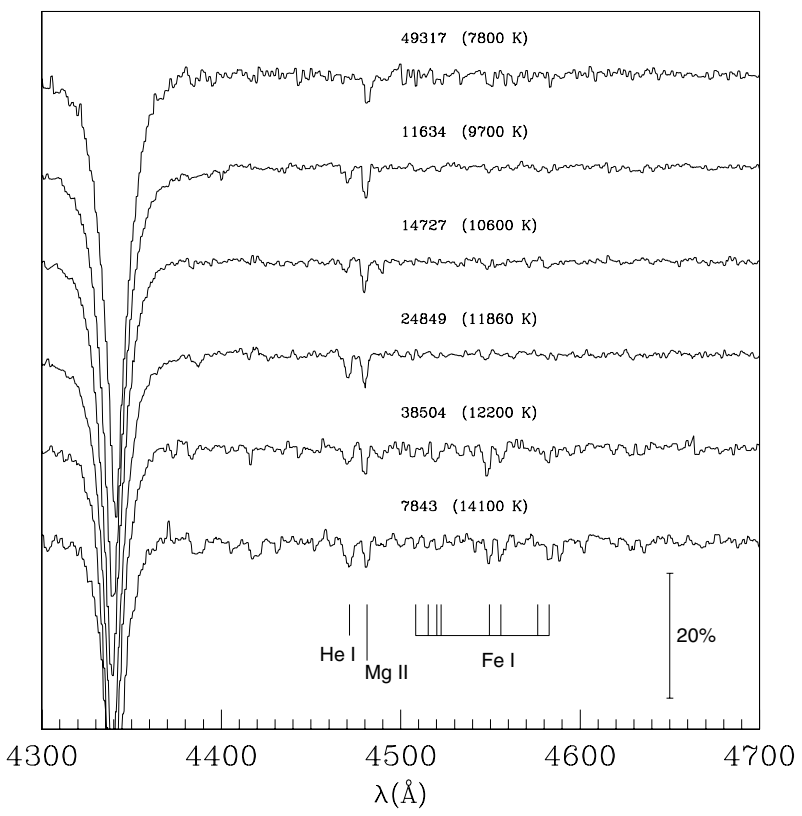

Fig. 3. A sample of spectra for stars across the Grundhal jump in the spectral range used to determine the metallicity of the model in the fitting procedure. Some of the most relevant Fe II are indicated with the $\mathrm{Mg}$ II and He I doublets that are the most important features in this spectral range.

Pace et al. 2006, NGC 2808). As the atmospheric metallicity has a non-negligible influence on the profiles of hydrogen and helium lines, we need to know if diffusion is active in a star to decide on the metallicity to fit our spectra. Therefore, in all spectra we carefully examined the region $4450 \AA$ to $4600 \AA$, where strong Fe II lines show up at $11000 \mathrm{~K}$ to $12000 \mathrm{~K}$. Past experience (Moehler et al. 1999, 2000, 2003) has shown that these lines are strong enough to allow detection even in mediumresolution spectra. In Fig. 3 we show this wavelength range for some spectra of stars across the Grundhal jump. Spectra showing evidence of iron lines or of being hotter than $14000 \mathrm{~K}$ (deduced from their position in the color-magnitude diagram) were fit with metal-rich $([\mathrm{M} / \mathrm{H}]=+0.5)$ model spectra, whereas stars below $14000 \mathrm{~K}$ with no evidence of strong iron lines were fit with metal-poor model $([\mathrm{M} / \mathrm{H}]=-1.5)$ spectra. In the cool star spectra, we kept the helium abundance fixed, as the helium lines in cool stars are rather weak, and the helium abundance should be close to solar anyway. During the fitting we verified that those helium lines predicted for these cool stars agree with the observed ones. We computed model atmospheres using ATLAS9 (Kurucz 1993) and used Lemke's version ${ }^{2}$ of the LINFOR program (developed originally by Holweger, Steffen, and Steenbock at Kiel University) to compute a grid of theoretical spectra that include the Balmer lines $\mathrm{H}_{\alpha}$ to $\mathrm{H}_{22}, \mathrm{He} \mathrm{I}$, and He II lines. The grid covered the range $7000 \mathrm{~K} \leq T_{\text {eff }} \leq 35000 \mathrm{~K}$, $2.5 \leq \log g \leq 6.0,-3.0 \leq \log \mathrm{He} \leq-1.0$, at metallicities of $[\mathrm{M} / \mathrm{H}]=-1.5$ and +0.5 . In Table 1 we list the results obtained from fitting the Balmer lines $\mathrm{H}_{\beta}$ to $\mathrm{H}_{10}$ (excluding $\mathrm{H}_{\epsilon}$ to avoid the $\mathrm{Ca}$ II H line) and, in the hot stars, also the He I lines $4026 \AA$, $4388 \AA, 4471 \AA$, $4921 \AA$ and the He II lines $4542 \AA$ and $4686 \AA$. IDs are from Momany et al. (2002). In cool star spectra the $\mathrm{He}$ abundance was kept fixed $(-1.00 \pm 0.00)$. Errors comes from the weighting procedure of two measurement for each star, and are multiplied by $\sqrt{3}$ but not corrected for possible underestimation. Masses were derived with Eq. (1). In last column peculiar stars are indicated, with the same symbols used in all figures.

To establish the best fit to the observed spectra, we used the routines developed by Bergeron et al. (1992) and Saffer et al. (1994), as modified by Napiwotzki et al. (1999), which employ a $\chi^{2}$ test. The $\sigma$ necessary for the calculation of $\chi^{2}$ is estimated from the noise in the continuum regions of the spectra. The fit program normalizes model spectra and observed spectra using the same points for the continuum definition.

For each star, the atmospheric parameters were measured independently in the two low-resolution spectra collected

\footnotetext{
2 For a description see http://a400. sternwarte.unierlangen.de/ ai26/linfit/linfor.html
} 
Table 1. Atmospheric parameters and derived masses for target stars.

\begin{tabular}{|c|c|c|c|c|c|}
\hline ID & $T_{\text {eff }}(\mathrm{K})$ & $\log g$ & $\log \frac{N(\mathrm{He})}{N(\mathrm{H})}$ & $M\left(M_{\odot}\right)$ & \\
\hline 14770 & $28400 \pm 300$ & $5.53 \pm 0.03$ & $-2.25 \pm 0.05$ & $0.55 \pm 0.04$ & \\
\hline 11634 & $9700 \pm 100$ & $3.39 \pm 0.05$ & $-1.00 \pm 0.00$ & $0.39 \pm 0.04$ & \\
\hline 14944 & $14500 \pm 100$ & $4.27 \pm 0.03$ & $-2.29 \pm 0.09$ & $0.51 \pm 0.04$ & \\
\hline 15026 & $8700 \pm 100$ & $3.08 \pm 0.05$ & $-1.00 \pm 0.00$ & $0.36 \pm 0.03$ & \\
\hline 16551 & $14500 \pm 100$ & $4.28 \pm 0.03$ & $-2.31 \pm 0.09$ & $0.51 \pm 0.04$ & \\
\hline 15395 & $25700 \pm 300$ & $5.58 \pm 0.03$ & $-2.54 \pm 0.05$ & $0.69 \pm 0.06$ & $\square$ \\
\hline 20919 & $8000 \pm 40$ & $2.91 \pm 0.03$ & $-1.00 \pm 0.00$ & $0.37 \pm 0.03$ & \\
\hline 18782 & $12100 \pm 100$ & $3.78 \pm 0.03$ & $-2.10 \pm 0.12$ & $0.47 \pm 0.04$ & \\
\hline 17941 & $24800 \pm 400$ & $5.02 \pm 0.03$ & $-1.98 \pm 0.05$ & $0.73 \pm 0.06$ & $*$ \\
\hline 20302 & 19100 & $4.87 \pm$ & $-1.78 \pm 0.03$ & 0.03 & \\
\hline 26756 & 104 & $3.55 \pm$ & $-1.00 \pm 0.00$ & 0.03 & \\
\hline 27181 & $13500 \pm 100$ & $3.96 \pm 0.03$ & $-1.98 \pm 0.09$ & 0.03 & $\Delta$ \\
\hline 24849 & 11860 & $4.09=$ & $-1.65 \pm 0.09$ & .03 & \\
\hline 27604 & $17600 \pm$ & $4.60=$ & $9 \pm 0.03$ & .08 & \\
\hline 28231 & $26900=$ & 5.5 & $4 \pm 0.03$ & .05 & प \\
\hline 26760 & 15600 & 4.42 & $3 \pm 0.07$ & .05 & \\
\hline 28554 & 2650 & 5.5 & $3 \pm 0.05$ & .06 & . \\
\hline 28693 & $28800 \pm 400$ & $5.56 \pm 0.03$ & $-3.26 \pm 0.02$ & 0.04 & \\
\hline 28947 & $22100 \pm 400$ & $5.17 \pm 0.03$ & $-1.84 \pm 0.02$ & $0.55 \pm 0.04$ & \\
\hline 4964 & $10740 \pm 100$ & $3.72 \pm 0.03$ & $-1.00 \pm 0.00$ & 0.60 & \\
\hline 49317 & 779 & $2.56=$ & $-1.00 \pm 0.00$ & 0.01 & \\
\hline 5455 & 26600 & $5.63=$ & \pm 0.02 & 0.06 & $\square$ \\
\hline 5487 & 2000 & 5.0 & $-1.60 \pm 0.02$ & 0.05 & \\
\hline 5134 & 152 & 4.3 & \pm 0.10 & 0.04 & \\
\hline 4672 & 252 & 5.3 & \pm 0.03 & .04 & \\
\hline 5201 & 2790 & 5.5 & $8 \pm 0.03$ & .03 & \\
\hline 5865 & 27800 & 5.53 & $7 \pm 0.05$ & .05 & $\square$ \\
\hline 7843 & 14100 & 4.07 & \pm 0.07 & .03 & $\Delta$ \\
\hline 6284 & 2720 & 5.41 & $-2.27 \pm 0.03$ & .04 & \\
\hline 10257 & 8800 & 3.06 & $-1.00 \pm 0.00$ & 0.03 & \\
\hline 10625 & 28700 & $5.67 \pm 0.03$ & $-1.84 \pm 0.03$ & 0.50 & \\
\hline 8672 & $30100 \pm 300$ & $5.73 \pm 0.03$ & $-2.90 \pm 0.09$ & $=0.04$ & \\
\hline 10711 & $27700 \pm 300$ & $5.63 \pm 0.03$ & $-2.28 \pm 0.05$ & 0.05 & $\square$ \\
\hline 11609 & $14300 \pm 100$ & $4.23 \pm 0.02$ & $-3.04 \pm 0.16$ & $0.51 \pm 0.04$ & \\
\hline 14664 & $8050 \pm 40$ & $3.02 \pm 0.03$ & $-1.00 \pm 0.00$ & $0.42 \pm 0.03$ & \\
\hline 14727 & $10600 \pm 100$ & $3.72 \pm 0.03$ & $-1.00 \pm 0.00$ & $0.81 \pm 0.06$ & \\
\hline 35186 & $10800 \pm 100$ & $3.73 \pm 0.03$ & $-1.00 \pm 0.00$ & 0.05 & \\
\hline 35662 & 12900 & $3.96=$ & .16 & 0.4 & \\
\hline 35499 & 1250 & 3.9 & $-2.09 \pm 0.12$ & 0.04 & \\
\hline 36242 & 12800 & 3.93 & $-2.03 \pm 0.10$ & $=0.03$ & \\
\hline 36480 & $22400 \pm 400$ & & $8 \pm 0.02$ & 0.4 & \\
\hline 36502 & 1230 & 3.8 & \pm 0.10 & 0.46 & \\
\hline 36830 & 27400 & 5.6 & \pm 0.05 & 0.6 & ( \\
\hline 38095 & $14300 \pm 200$ & $4.03 \pm 0.03$ & $-1.93 \pm 0.05$ & 0.02 & $\Delta$ \\
\hline 38087 & $27300 \pm 300$ & $5.53 \pm 0.03$ & $-2.18 \pm 0.05$ & 0.63 & ח \\
\hline 32470 & $10620 \pm 90$ & $3.59 \pm 0.03$ & $-1.00 \pm 0.00$ & 0.03 & \\
\hline 28695 & $9600 \pm 100$ & $3.35 \pm 0.07$ & $-1.00 \pm 0.00$ & 0.04 & \\
\hline 38504 & $12200 \pm 100$ & $3.90 \pm 0$ & $-2.13 \pm 0.10$ & 0.50 & \\
\hline 39008 & $30900 \pm 300$ & $5.55 \pm 0.03$ & $-2.29 \pm 0.05$ & $0.53 \pm 0.05$ & \\
\hline 38889 & $12700 \pm 200$ & $3.94 \pm 0.05$ & $-2.00 \pm 0.17$ & $0.47 \pm 0.04$ & \\
\hline 38963 & $9000 \pm 200$ & $3.16 \pm 0.09$ & $-1.00 \pm 0.00$ & $0.32 \pm 0.04$ & \\
\hline
\end{tabular}

during the observing run. The final results given in Table 1 are the weighted means of these values, weighted by the inverse errors provided by the fit procedure. The tabulated errors are the results of the weighted mean procedure applied to the pair of independent measurements obtained by the fit of the two spectra for each star. We multiplied the errors given by the fitting routine by $\sqrt{3}$ because, when rebinning, we oversampled the spectra by a factor of three with respect to the dispersion, while the fitting procedure assumes each pixel is independent of the others. The errors in each fit of the observed spectrum with the model spectrum were derived from the $\chi^{2}$ of the fit itself (see Moehler et al. 1999, for more details). These errors are obtained under the assumption that the only error source is the statistical noise (as derived from the continuum of the spectrum). However, Napiwotzki (priv. comm.) noted that the routine underestimates this statistical error by a factor of 2-4.

In addition, errors in the normalization of the spectrum, imperfections of flat field/sky background correction, etc. may produce systematic rather than random errors, which are not represented well by the error obtained from the fit routine. Masses were calculated from the previously measured atmospheric parameters in two ways. First we used the equation

$\log \frac{M}{M_{\odot}}=$ const. $+\log g+0.4\left((m-M)_{V}-V-V_{\text {th }}\right)$

where $V_{\text {th }}$ is the brightness at the stellar surface as given by Kurucz (1992). In addition we also calculated masses through the equation

$\log \frac{M}{M_{\odot}}=\log \frac{g}{g_{\odot}}-4 \log \frac{T}{T_{\odot}}+\log \frac{L}{L_{\odot}}$

obtained from basic relations, assuming $T_{\odot}=5777 \mathrm{~K}$ and $\log g_{\odot}=4.4377$. In the last term we expressed the luminosity in terms of absolute magnitude and bolometric correction $\mathrm{BC}$ through the relation

$\log \frac{L}{L_{\odot}}=-\frac{M_{V}+B C-4.74}{2.5}$.

The bolometric correction for each target star was derived from effective temperatures using the empirical calibration of Flower (1996). In both equations we used photometric data from Momany et al. (2002), and we adopted an apparent distance modulus $(m-M)_{V}=13.17$ and $E_{B-V}=0.04$ as the mean value of the determination of Renzini et al. (1996), Reid (1997, 1998) and Gratton et al. (1997). Results from both equations are plotted separately in Fig. 7. Errors on masses were derived from propagation of errors, including the $\sqrt{3}$ factor for errors on $T_{\text {eff }}$ and $\log g$. We also assumed an error of $0.1 \mathrm{mag}$ on the photometric quantities used in the equations, such as distance modulus, magnitude, $V_{\text {th }}$, and bolometric correction.

\section{Results}

\subsection{Temperatures and gravities}

Our results on atmospheric parameters are plotted in Fig. 4, where the position of the target stars in the $T_{\text {eff }}-\log g$ space is compared with theoretical models. The agreement between the measured parameters and the expectaction from the models is good, and all stars but a few exceptions fall between the theoretical zero-age HB (ZAHB) and the terminal-age HB (TAHB) for normal He-content $(Y=0.24)$ stars.

We note a group of hot stars $\left(T_{\text {eff }} \approx 27000 \mathrm{~K}\right)$ for which the measured surface gravities seem to be systematically higher than expected for normal He content stars. Another group of three cooler stars $\left(T_{\mathrm{eff}} \approx 14000 \mathrm{~K}\right)$ shows gravities that are too low and lies above the TAHB. Though these differences are marginal in the $T_{\text {eff }} \log g$ plot, they appear more significant when we consider the masses (see Fig. 7). It is worth noticing in Fig. 4 that they are all reproduced better by models with enhanced helium abundance $(Y=0.33)$.

There is an additional anomalous star at $T_{\text {eff }} \approx 25000 \mathrm{~K}$, which has a significantly smaller surface gravity than expected. This star (marked as asterisk in all the figures in this paper) also has an anomalous position in the color-magnitude diagram and 


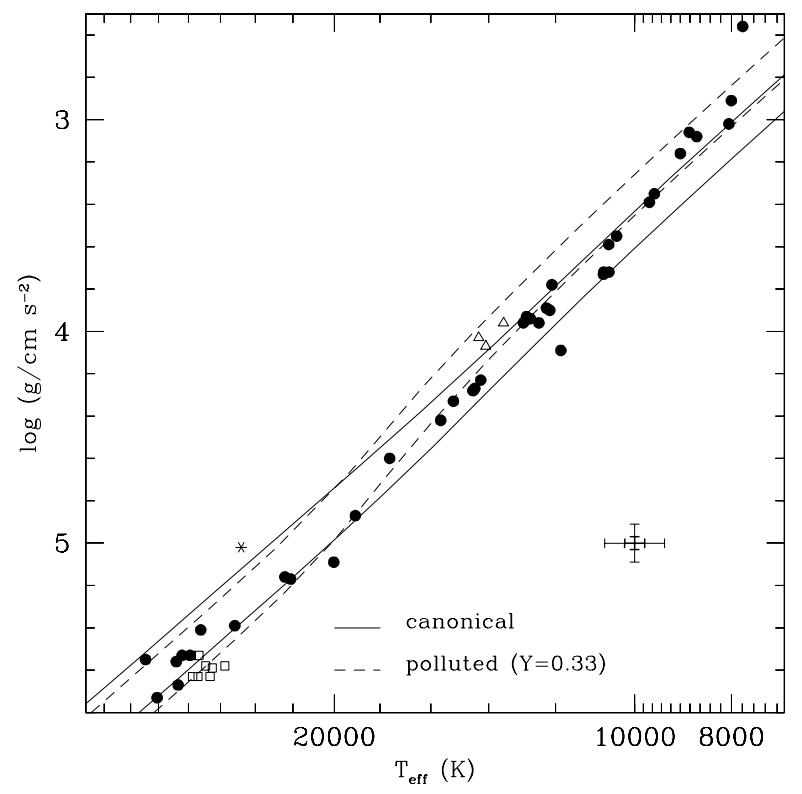

Fig. 4. Temperatures and gravities of our program stars. All stars hotter than $12000 \mathrm{~K}$ or showing evidence of iron enrichment are fitted with model atmospheres of super-solar metallicity $([\mathrm{M} / \mathrm{H}]=+0.5)$. Cooler stars showing no evidence of iron enrichment are fitted with metal-poor model atmospheres $([\mathrm{M} / \mathrm{H}]=-1.5)$. The solid lines mark the zero-age (ZAHB) and terminal-age (TAHB) loci of canonical HB tracks $(Y=$ 0.24 ) for $[\mathrm{M} / \mathrm{H}]=-1.54$ (see Moehler et al. 2003, for details). These loci define the region within which the HB models spend $99 \%$ of their HB lifetime. Dashed lines represent the ZAHB and the TAHB of helium enriched $(Y=0.33)$ stars. Error bars are omitted for clarity, but the typical size of the errors as derived from the $\chi^{2}$ fit is indicated. The thick error bars indicate the formal error as in Table 1, and the larger thin error bars indicate three times the previous value for hotter stars, approximately $1000 \mathrm{~K}$ and $0.1 \mathrm{dex}$, which is probably a better estimate of real errors (see text for details).

will be discussed later. All the anomalous stars have radial velocities compatible with the radial velocity of NGC 6752, as can be seen in Table 1 of Paper I. Therefore they can be considered probable cluster members, but it must be noted that radial velocity alone is not an unambiguous proof of membership in this cluster. In fact, at the galactic coordinates of NGC 6752 $\left(l=336^{\circ}, b=-26^{\circ}\right)$, a certain amount of field contamination is expected, and its radial velocity is unremarkable compared to field disk stars (-27.9 $\mathrm{km} \mathrm{s}^{-1}$, Harris 1996).

We compared the derived effective temperatures with those measured in Moehler et al. (2000) for HB stars in the same cluster. The temperatures of both data sets are plotted as a function of color in Fig. 5. The results agree very well. There is a small offset of our data toward lower temperatures, but it cannot be considered significant because it is much smaller $(\leq 500 \mathrm{~K})$ than formal errors. We also have nine stars in common with Moehler et al. (2000). In Fig. 5 we plot the differences between the two works in derived $\log g$ and masses. Within the errors, the results of the two investigations are consistent, as was expected due to the similarities between the two works: data reductions are almost identical, and measurement procedures and model atmospheres are the same.

\subsection{Masses}

In Fig. 7 we plot the masses we derived for our target stars as a function of effective temperatures, with both Eqs. (1) and (2).

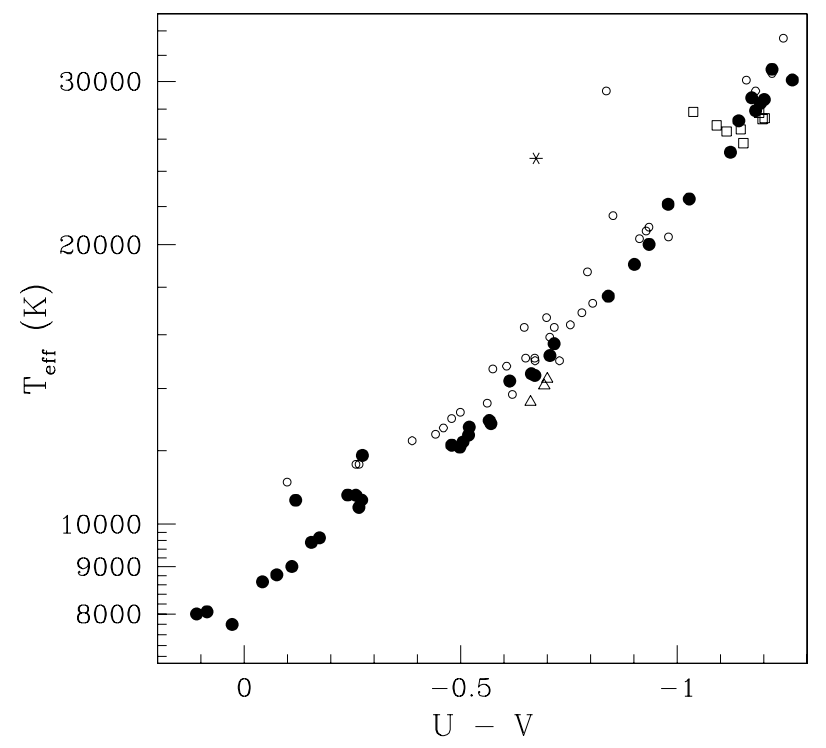

Fig. 5. Measured effective temperatures as a function of colors derived in this work (full circles, and triangles and squares follow the notation in previous plots) and in Moehler et al. (2000) (small empty circles). The adopted data from Moehler et al. (2000) are the ones obtained with metal-poor model atmospheres (their Table 2) for stars with $T_{\text {eff }} \leq 11000 \mathrm{~K}$ and with metal-rich model atmospheres (their Table 5) for hotter stars.
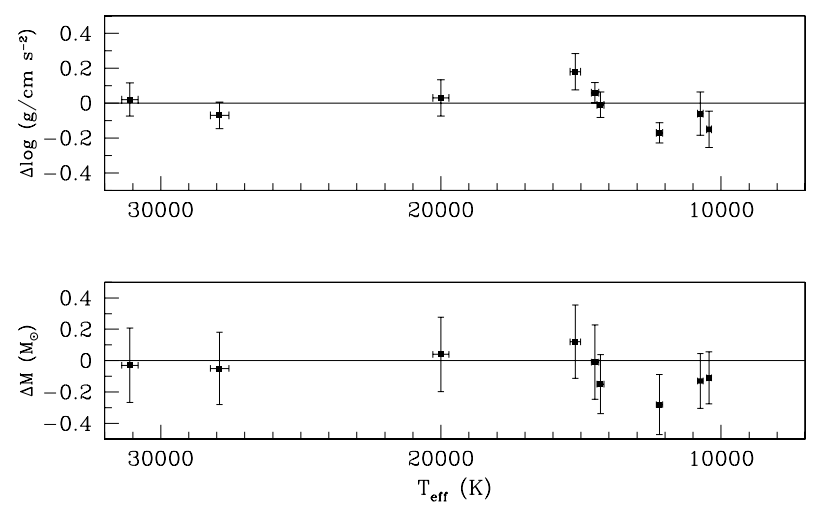

Fig. 6. Differences in surface gravities and derived masses between this work and Moehler et al. (2000) for the nine stars in common. The errors are the quadratic sum of the ones for each set of data. The difference is in the sense (ours) - (M00).

Here the problems and the differences between observed and expected values become evident. We note that both equations give very similar results, and the differences are negligible in all but the temperature range $12000-15000 \mathrm{~K}$. In this interval the masses calculated with Eq. (2) show better agreement with theoretical expectations because they are on average $0.05 M_{\odot}$ higher, but it is not enough to correct the general tendency of an underestimation of the derived masses for $T_{\mathrm{eff}} \leq 15000 \mathrm{~K}$. We discuss this problem in more details in subsequent sections.

Here we note that, although the plotted points in this temperature range are closer to the theoretical line in the lower panel of Fig. 7, they are not scattered around it, but systematically below it, as in the upper panel. This indicates that the problems concerning derived masses cannot be ascribed only to either the theoretical $V_{\text {th }}$ values used in Eq. (1) or to empirical BCs used in Eq. (2), because both equations lead to the same underestimation independently. 

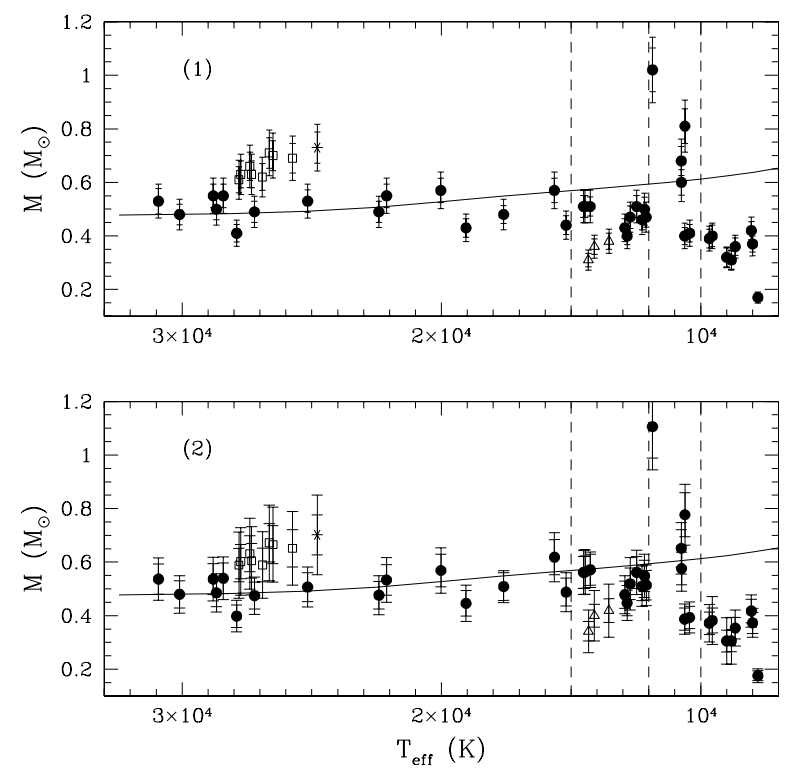

Fig. 7. Estimated masses of target stars as a function of effective temperature. Different symbols are used to distinguish between groups of stars that behave differently, as indicated in the text. Also the theoretical HB (Moehler et al. 2003) is indicated. Error bars on temperatures are omitted for clarity, but are within an order of magnitude of full points. Errors on masses are obtained from the propagation of errors on temperature and gravities, this error bars referring to the formal errors given in Table 1, and larger ones assuming errors of $1000 \mathrm{~K}$ and 0.1 dex respectively, which are probably more realistic estimates of real errors. Vertical dashed lines divide the temperature intervals analyzed in the text. Upper panel: masses derived from Eq. (1). Lower panel: masses derived from Eq. (2).

The problem cannot be attributed to the $V$ magnitudes from Momany et al. (2002) that we use to derive the masses. When calibrating photometric data, we all refer to the standard $U B V$ Johnson filters and calibrate with respect to them. However, the study by Momany et al. (2003) basically shows that no 2 filtersystems are the same (see their Fig. 2) and that small differences in the bandpass of the employed filters (at any telescope) may persist. Momany et al. also show that such "small" differences can eventually give rise to un-physical features. and we are aware that the WFI@2.2 m V filter has a red cutoff at $\lambda \sim 5900 \AA$ with respect to the standard $V$ filter. Moreover, the WFI@2.2 m instrumental $v$ magnitudes were calibrated with their respective $(b-v)$ colors, thus employing the WFI@2.2 $\mathrm{m} B$ filter with a redder effective central wavelength. Nevertheless, it remains quite hard to explain the derived low masses in terms of photometric calibration uncertainties. Indeed, to derive masses that match theoretical expectations, we estimate that the $M_{V}$ magnitudes need to be increased by $\sim 0.4$ in the $12000-15000 \mathrm{~K}$ range, and decreased by a similar amount for the hot outliers. Clearly, this discrepancy is too large to be attributed to photometric calibration uncertainties.

Because of the lack of noticeable differences between the panels in Fig. 7, we discuss the results without distinguishing between the two methods to derive masses. Instead, we find it appropriate to divide the sample to three different ranges of temperatures.

\subsubsection{Masses: cool stars ( $\left.T_{\text {eff }} \leq 12000 \mathrm{~K}\right)$}

The derived masses of stars cooler than $10000 \mathrm{~K}$ are systematically too low. All of these stars are offset from the HB towards lower gravities in Fig. 4. Moehler et al. (2003) observed the same effect for HB stars in M13 and excluded these stars from their analysis. It is worth noticing that Moehler et al. (2000) find masses in this range of temperatures in good agreement with the expected value for stars in the same cluster, but Moehler \& Sweigart (2006) in NGC 6388 find masses that are too low, as we do with the same instruments and set-up as in the present work (UT4+FORS2 and grism B600). They consider that atmospheric parameters for these stars are probably not trustworthy, because of the presence of metallic lines not considered in the fitting procedure and not clearly observable due to low resolution. In the case of NGC 6752, metal lines offer less explanation because this cluster is more than a factor of 10 more metal-poor than NGC 6388. However because of the coincidence observed, it can be hypothesized that, whatever the cause, it could somehow be enhanced by the instrumentation and set-up used. Moehler et al. (2003) find too low mass for the star they observed in this temperature range, in a cluster of similar metallicity (M3) but with different instrumentation. This excludes the possibility that the underestimation was only due to instrumental effect with this setup, and the problem of the masses too low for $T_{\text {eff }} \leq 10000 \mathrm{~K}$ remains completely open. The calculated masses for stars between 10000 and $12000 \mathrm{~K}$ better agree with theoretical values although the scatter is quite high.

\subsubsection{Masses: intermediate temperature stars $\left(12000 \leq T_{\text {eff }} \leq 15000 \mathrm{~K}\right)$}

For higher temperatures, as the radiative levitation sets in, masses are constantly underestimated. This is a well-known result that is clearly present in our data. In fact, even if the errors on the masses of the single stars are high, Fig. 7 clearly shows that our derived masses in the $12000 \leq T_{\text {eff }} \leq 15000 \mathrm{~K}$ interval are systematically lower than predicted. Our results are also in agreement with the ones of Moehler et al. (2000), and the difference that we find between observed and expected masses is of the same order of magnitude as in their work. We then confirm their conclusions that the use of models with higher metallicity in the fitting procedure, to account for radiative levitation, can only partially reduce the discrepancy.

There is a group of three stars located at $13000<T_{\text {eff }}<$ $14000 \mathrm{~K}$ with particularly low masses and low gravities. These stars are indicated in all of the figures as empty triangles. These stars are also separated well in the color-magnitude diagram (Fig. 1), as they are systematically brighter than the others. It must be emphasized that, although we relied on photometric data to derive masses, the difference in masses cannot be ascribed to the difference in magnitude, since they go in opposite directions: all other parameters being the same, brighter stars should show higher masses, and not lower, as can be deduced by Eq. (1). These anomalous masses are probably due to the very low surface gravities, low enough to give underestimated masses, even if the stars are brighter than their neighbors in the color-magnitude diagram. Therefore, for some reason, these stars have different luminosities and a different spectrum than other HB stars of similar temperatures. In this temperature range, there is another star (\#18782) brighter than the average HB in Fig. 1 and outside (above) the theoretical tracks in Fig. 4. Nevertheless its calculated mass is not noticeably low with respect to other stars with similar temperatures, so we did not point it out with a different symbol. We do take it into account in the later discussion. 


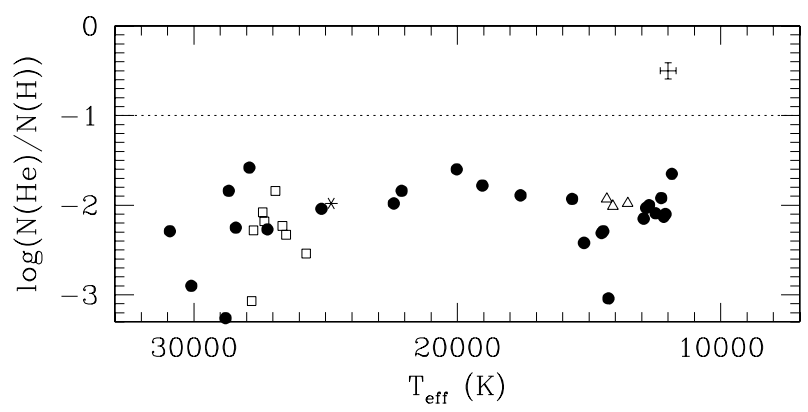

Fig. 8. Derived helium abundances as a function of effective temperatures for target stars. Different symbols are used to distinguish between groups of stars that behave differently, as indicated in the text. The dotted line indicates solar value, and the typical errorbars $(0.09$ dex in $\log (N((\mathrm{He}) / N(\mathrm{H}))$ and $300 \mathrm{~K}$ in temperature) are indicated in upper right. For stars cooler than the Grundhal jump the abundance was kept fixed to solar value in the fitting procedure, and they are omitted in the plot.

\subsubsection{Masses: hot stars ( $\left.T_{\text {eff }} \geq 15000 \mathrm{~K}\right)$}

For increasing temperatures, the measured masses again generally agree with theoretical values, as also found by Moehler et al. (2000) in the same cluster. Nevertheless, also among hotter stars there is a group of stars that behave differently from the general trend. These objects are indicated as empty squares in all the plots, and for them the derived masses are systematically higher than expected. As shown in Fig. 1, they are fainter than the bulk of HB stars at the same temperature. Again, Eq. (1) would imply lower masses for fainter stars, so this is not caused by how we derived our masses: either these objects are intrinsically more massive or they are both photometrically and spectroscopically different from other stars at the same temperature.

\subsection{Helium abundances}

The derived He abundances are plotted in Fig. 8 as a function of effective temperatures, where only stars hotter than Grundhal jump are considered because, for cooler ones, He abundance was not measurable, hence kept fixed to solar value. All stars show helium depletion to a different extent, but no trend with temperature is visible.

The three stars indicated as triangles, which show particularly low gravities and/or masses, on average also show a higher helium abundance than do other stars at the same temperature, though they agree with values for stars at different temperatures - from cooler ones up to $20000 \mathrm{~K}$. On the other hand, no clear trend is visible for hot stars with anomalous measured masses, which show helium abundances in agreement with other hot stars.

\subsection{Star \#17941}

Star \#17941 is very peculiar and its measured atmospheric parameters are highly uncertain. This object is indicated with an asterisk in all the figures.

Its radial velocity, as measured in Paper I, indicates that it is a probable cluster member. In the color-magnitude diagram it is located below the HB at fainter magnitudes and/or redder color. Its $B-V$ color indicates an effective temperature around $14000 \mathrm{~K}$, which is also confirmed by its intermediateresolution spectrum (Paper I), which shows the typical features of an HB star with this temperature, in particular, a high quantity

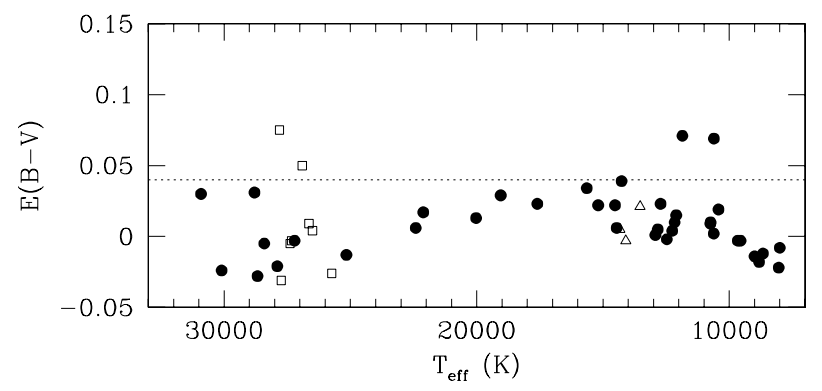

Fig. 9. Estimated $E_{(B-V)}$ reddening values for target stars. The dotted line indicates the value $E_{(B-V)}=0.04$, which is the mean value of the determination of Renzini et al. (1996), Reid (1997, 1998), and Gratton et al. (1997).

of metallic lines due to radiative levitation. Actually, its spectrum is very similar to the ones of other stars in this temperature range. On the contrary, from the measurements on lowresolution spectra, it comes out to be a much hotter HB star $(24800 \mathrm{~K})$. Also the surface gravity is peculiar, being particularly low. Even more puzzling, and at variance with the behavior of all other peculiar stars already analyzed, we find a higher mass than expected even if its surface gravity is lower than canonical models.

We analyzed the data reduction of these spectra in details, trying different solutions for spectra reduction and extraction, with no significant changes in the derived parameters. We found no evidence of light contamination by a nearby star, both when analyzing the slit images and in the field images acquired before exposures. We did not find any indication of any companion, not even among the spectral features observed at intermediate resolution, although this could not be indicative because the resolution itself is not very high $(1.2 \AA)$, and the great quantity of metallic lines could easily hide fainter features from a companion.

\subsection{Reddening}

With the derived effective temperatures and gravities, we estimated the color excess $E(B-V)$ that could be deduced from these atmospheric parameters. We used the Kurucz ATLAS9 (Kurucz 1993) photometry table for the same metallicity as was used in fitting procedure, and for each star we interpolated the $B-V$ values in the grid to get the expected values. Then we calculated the color eccess $E(B-V)$ by comparing this theoretical color with the observed one from Momany et al. (2002) photometry. The results are plotted in Fig. 9.

The computed reddening is constantly too low compared to literature values. In fact the mean value of Renzini et al. (1996), Reid (1997, 1998), and Gratton et al. (1997) that we also used in Eq. (1) is $E(B-V)=0.04$, and it is in fair agreement with the more recent measurement by Gratton et al. (2005). We obtain $E(B-V)=0.016$ as the mean value for our stars, with no particular trend with temperature.

It should be noted that the WFI@2.2 m bandpasses differ significantly from the standard Johnson-Cousins ones (Momany et al. 2002), and an error of $0.02 \mathrm{mag}$ in color cannot be excluded. However, Gratton et al. (2005) use the same photometric data and obtain reddening estimates in perfect agreement with previous works among turn-off stars in the same cluster. The main difference is that they estimated temperatures fitting $\mathrm{H}_{\alpha}$ profiles in high-resolution spectra, a totally different procedure from the one adopted here. Therefore, we are forced to conclude 
that the offset observed here could probably point to a systematic underestimate of the temperature along the HB. Of course, the problem could reside in the theoretical colors themselves. In fact, empirical calibrations of $T_{\text {eff-color relations show that syn- }}$ thetic colors from model atmospheres calculated with ATLAS9 have difficulty properly reproducing observations (Ramırez \& Meléndez 2005; Sekiguchi \& Fukugita 2000), a problem also pointed out in the theoretical investigation by Castelli et al. (1997). Unfortunately, all the cited empirical calibrations are limited in the cool temperature range (spectral type G-K) of main sequence or giant stars, and there is a general lack of HB colortemperature, model-independent calibrations in the literature. In conclusion, it is not possible to guess to what extent ATLAS9 models could lead to a systematic error in temperature.

The stars that show peculiar masses show no particular behavior in Fig. 9. Since reddening estimates are very sensitive to temperature but should hardly change for small variations of surface gravity, this can indicate that their derived temperature are not peculiar, and their strange behavior is only due to an unusual surface gravity (either real or as a consequence of other effects unaccounted for).

\section{Discussion}

We assumed LTE in our model spectra, as usual in this kind of analysis, but it is becoming evident that non-LTE effects could affect the results for hotter stars and lead to erroneous atmospheric parameters, as pointed out by Przybilla et al. (2006). In their preliminary results they show that a hybrid NLTE analysis can provide a much better fit of $\mathrm{H}$ and $\mathrm{He}$ lines, but the differences in atmospheric parameters relative to results assuming LTE are only slightly larger than our real uncertainties (on the order of $1000 \mathrm{~K}$ in $T_{\text {eff }}$ and $0.1 \mathrm{dex}$ in $\log g$ ). Considering that errors in Table 1 are underestimated by a factor of at least 2-4, as already discussed, the influence of neglecting NLTE effects is relatively small. Even more important, it should affect all stars in the same way at a given temperature (NLTE analysis provides higher temperatures and gravities than the LTE one), so it should not cause any systematic difference for stars at approximately the same temperature. However, adding $1000 \mathrm{~K}$ in $T_{\text {eff }}$ and $0.1 \mathrm{dex}$ in $\log g$ strongly increases the derived masses as calculated from Eq. (1). The change in temperature is reflected in a decrease of $\approx 0.1 \mathrm{mag}$ for $V_{\mathrm{th}}$, which is quite insensitive to gravity. Masses are then higher by about $0.2 M_{\odot}$ in the high temperature range. The effect on masses derived from Eq. (2) is much smaller, approximately half the previous one, but again in the sense of increasing masses. While this would alleviate our problems between $12000 \mathrm{~K}$ and $15000 \mathrm{~K}$, there is no reason to assume that NLTE effects would be limited to this temperature range.

We also assumed solar abundance ratios in the models. This is necessarily a crude approximation in order to take into account the enhanced abundancies of heavy elements due to radiative levitation. Detailed studies both in the proximity of the Grundhal Jump (Behr et al. 1999; Behr 2003) and among hotter HB stars (Edelmann et al. 2001) show that the atmospheric metal abundances in the presence of radiative levitation do not usually follow solar ratios: the elements are affected to different extents and some are even not enhanced at all. The pattern of abundance ratios and its effect on the atmospheric structure are still poorly understood. The approximation of solar abundance ratio could introduce a systematic effect in parameter determination to an unknown extent, and in principle stars with different abundance patterns (due to different formation history) could behave differently in our analysis. Eitherway, there are indications
(Edelmann et al. 2006) that, at least for EHB stars, the surface abundance pattern could almost be independent of stellar parameters, so any systematic effects, if relevant, should be the same in a wide range of temperatures and gravities. This might not be the case for cooler stars. In fact, Pace et al. (2006) find a clear trend toward increasing heavy elements abundance with effective temperatures across the Grundhal jump $\left(10000 \leq T_{\text {eff }} \leq 14000 \mathrm{~K}\right)$. Therefore, at least in this temperature range, the deviations from solar abundance ratio (and then the differences with respect to the models) should be increasing with effective temperature. This could help for explaining our results, but the extent of the consequences on derived parameters is unknown, and in Fig. 7 a clear trend with temperature in this range is not visible.

Our results for the three peculiar stars at $14000 \mathrm{~K}$ appear to be compatible with the hypothesis of helium-enrichment (as shown in Fig. 4). However, in this case the low surface gravity is directly linked to the higher luminosity as shown by Catelan (2005) and Sweigart \& Catelan (1998). The masses derived from effective temperatures, surface gravities, and luminosities should therefore be well above $0.5 M_{\odot}$. The low masses derived here suggest that the low gravities are at least partly spurious. The same reasoning seems to exclude the hypothesis that these stars are an evolving or evolved object, as could be guessed looking at their position in the color-magnitude diagram. In fact, in this case again, the higher luminosities would compensate for the lower gravities, and the masses should agree with expectations. This is what we observe for star \#18782 at $U-V \approx-0.5$, which shows both brighter luminosity and lower gravity than other stars, but this is not peculiar for its calculated mass. Therefore we suppose that helium-enrichment or evolutionary effects can be considered good hypothesies that explain our observations for this star.

It must be noted that the measured helium abundance cannot be properly disentangled from measured gravities because both have been measured with the same fitting procedure. Helium abundances are mainly measured from He lines, but as investigated by Moehler \& Sweigart (2006), varying the helium abundance directly changes the width of Balmer lines, in the sense that higher helium abundances should produce broader lines. This rules out the possibility that low gravities are produced by an unnoticed helium enrichment, but indicates that underestimating gravity and overestimating helium abundance are two correlated effects. That these stars are systematically brighter prevents concluding that the anomalous parameter comes only from this weakness in the fitting procedure. These stars must be intrinsically different from fainter ones.

Probably the best explanation is that our results indicate enhanced stellar winds. In fact Vink \& Cassisi (2002) indicate that a non negligible wind should be expected for HB stars. They also show how it can change Balmer line profiles in such a way as to mimic a lower surface gravity. There is still a lack of detailed models that take the presence of wind into account, making it necessarily unaccounted for in our fitting procedures. Then, the derived low surface gravities could result from this fault. The wind scenario is corroborated by the anomalous stars being brighter, so an enhanced stellar wind could be expected. Moreover, a wind could possibly counteract the diffusion, which reduces the atmospheric helium abundance observed in subdwarf B stars to sub-solar values and thus justifies the higher helium abundances we measure.

It is harder to find a good explanation for our results on the eight hot peculiar stars indicated as open squares in all plots. They were first spotted in Fig. 7 for their abnormally high masses, and then individuated in the color magnitude diagram as 
being fainter and/or redder than other stars observed in the same color-magnitude range.

In this case we can assume that spectroscopically derived masses and photometric properties are independent. It is not generally true because magnitudes were used in the mass calculation, but as we saw, high masses cannot be ascribed to fainter magnitudes, and then the two effect cannot be related. The cause of peculiar masses must be other than photometric data, and we would expect these stars to be randomly distributed among the EHB population. Under this assumption, we performed a twodimensional Kolmogorov-Smirnov (KS) test to explore the possibility that these stars are also photometrically peculiar. The two-dimensional version of the KS-test is not mathematically well-defined, but we adopted the algorithm of Peacock (1983), which gives reliable results for all the cases of practical interest. We compared the distribution of the eight stars in the colormagnitude diagram with the whole population of stars in the range $-1.25 \leq(U-V) \leq-1$ and $17 \leq V \leq 17.5$. The test reveals that there is a $8.4 \%$ probability that the analyzed sample is randomly drawn from the test distribution. This result is clearly not conclusive, but indicates that with high probability $(91.6 \%)$ the eight hot peculiar stars indeed show photometric peculiarities and could belong to a sub-population of stars different from the brighter and bluer bulk of EHB star population. It can be excluded that this result stems from a selection effect in the target selection, because the same test, when applied to all 13 spectroscopically observed stars in this temperature range, gives a $25 \%$ probability of this sample being drawn from the test distribution.

For the eight hot peculiar stars, the helium enrichment hypothesis is a possible explanation. In fact, in the $T_{\text {eff }}-\log g$ plot these stars agree better with polluted models and, according to calculations by Sweigart \& Catelan (1998), an increased helium abundance in the high temperature regime implies higher gravity. However, in this case their overly high masses again put this explanation in question, because higher gravities and lower magnitudes would balance out and lead to normal derived masses.

As these stars are redder than their neighbors in the colormagnitude diagram, they might be binaries. In this case, contamination by the light of a cool companion could affect the parameter determination, and additional light would increase any derived mass. We therefore analyzed the spectra looking for signatures of the presence of cool companions, both in the lowresolution spectra used in this work and the higher resolution ones from Paper I, focusing on features typical of G-K type stars, such as the G-band and the MgIb triplet. We found no evidence of companions, except for star \#5865, which shows a strong MgI triplet, unusual for such a hot star (see Fig. 10). Although this could indicate a binary system, it is just an isolated case, and the failure to individuate these features in all the other seven stars puts the hypothesis of binarity for these peculiar hot stars in doubt. In addition we did not find any indication in Paper I of radial velocity variations for any of these stars.

In principle, we cannot exclude that their masses are actually higher, but it appears to be quite improbable and hard to explain. It is simpler to think that the gravities are overestimated by fitting procedures, but even this conclusion has important implications. As a consequence, we must deduce that models are unable to describe these objects and their spectra properly. Some difference between the model and the real star should be responsible for unreliably measured masses. But the same models give results in perfect agreement with theoretical expectations for "normal" stars, therefore the "peculiar" ones must be intrinsically different from the main population. This conclusion is strongly

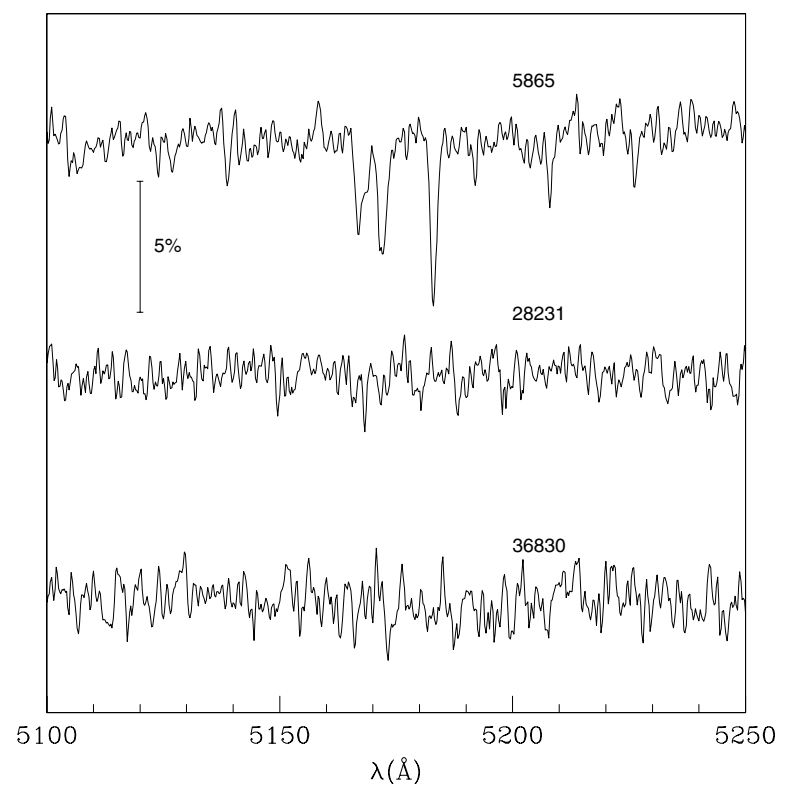

Fig. 10. A section of the spectrum of star \# 5865 where the MgI triplet is clearly visible. The spectra of two other peculiar hot stars are plotted for comparison. The spectra have been obtained for each star by summing the high-resolution ones used in Paper I, after correcting for RV shifts.

corroborated by the color-magnitude diagram, where peculiar stars seem to be fainter and/or redder than the others.

We point out that the hot peculiar stars lie in concomitance of the Momany Jump (Momany et al. 2002), a feature discovered first in NGC 6752 and then also individuated in other clusters (Momany et al. 2004). The Jump is located around $T_{\text {eff }}=$ $23000 \mathrm{~K}$ and is characterized by a sudden increase in luminosity of the HB stars. As suggested by the author of the discovery paper, the presence of the jump could be due to deep changes in atmospheric structure at this critical temperature, with the onset of radiative levitation and changes in chemical abundances. We find no good explanation for our results in this direction, since we would expect brighter stars (and not fainter) to be deviating with respect to the models, but it is a coincidence that probably should be investigated further.

\section{Conclusions}

We measured atmospheric parameters $\left(T_{\text {eff }}, \log g, \log \frac{N(\mathrm{He})}{N(\mathrm{H})}\right)$ and derived masses for $51 \mathrm{HB}$ stars in NGC 6752. Our results agree in general with previous studies, but not always with theoretical expectations. Like previous works in literature, we find masses that are too low for cool $\left(T_{\text {eff }} \leq 10000 \mathrm{~K}\right)$ and intermediatetemperature $\left(12000 \leq T_{\text {eff }} \leq 15000 \mathrm{~K}\right)$ stars. We find two groups of stars deviating with respect to the general behavior of the sample. The first group at $14000 \mathrm{~K}$ shows masses that are too low, for which we consider a weak stellar wind (unaccounted for in the model spectra) as the most probable explanation. The second group at about $27000 \mathrm{~K}$ shows too high masses, for which we found no good explanation. These stars most probably (91.6\%) do not belong to the general distribution of EHB stars in the color-magnitude diagram. We conclude that atmospheric models, successful in reproducing the other EHB stars (for which we obtain masses in agreement with expectations), are inadequate for these peculiar ones.

Acknowledgements. C.M.B. acknowledges Universidad de Chile graduate fellowship support from programs MECE Educación Superior UCH0118 and 
Fundación Andes C-13798. We want to thank the staff at the La Silla Paranal Observatory for their support during our observations.

\section{References}

Behr, B. B. 2003, ApJS, 149, 67

Behr, B. B., Cohen, J. G., McCarthy, J. K., \& Djorgovski, S. G. 1999, ApJ, 517, L135

Behr, B. B., Cohen, J. G., \& McCarthy, J. K. 2000a, ApJ, 531, L37

Behr, B. B., Djorgovski, S. G., Cohen, J. G., et al. 2000b, ApJ, 528, 849

Bergeron, P., Saffer, R. A., \& Liebert, J. 1992, ApJ, 394, 228

Castelli, F., Gratton, R. G., \& Kurucz, R. L. 1997, A\&A, 318, 841

Catelan, M. 2005, in Resolved Stellar Populations, ASP Conf. Ser., in press [arXiv: astro-ph/0507464]

Catelan, M., Borissova, J., Sweigart, A. V., \& Spassova, N. 1998, ApJ, 494, 265

Edelmann, H., Heber, U., \& Napiwotzki, R. 2001, Astron. Nachr., 322, 401

Edelmann, H., Heber, U., \& Napiwotzki, R. 2006, Baltic Astron., 15, 103

Fabbian, D., Recio-Blanco, A., Gratton, R. G., \& Piotto, G. 2005, A\&A, 434, 235

Faulkner, J. 1966, ApJ, 144, 978

Ferraro, F. R., Paltrinieri, B., Pecci, F. F., Rood, R. T., \& Dorman, B. 1998, ApJ, 500,311

Flower, P. J. 1996, ApJ, 469, 355

Glaspey, J. W., Demers, S., Moffat, A. F. J., \& Shara, M. 1985, ApJ, 289, 326

Glaspey, J. W., Michaud, G., Moffat, A. F. J., \& Demers, S. 1989, ApJ, 339, 926

Gratton, R. G., Fusi Pecci, F., Carretta, E., et al. 1997, ApJ, 491, 749

Gratton, R. G., Bragaglia, A., Carretta, E., et al. 2005, A\&A, 440, 901

Grundahl, F., Catelan, M., Landsman, W. B., Stetson, P. B., \& Andersen, M. I. 1999, ApJ, 524, 242

Hamuy, M., Suntzeff, N. B., Heathcote, S. R., et al. 1994, PASP, 106, 566

Harris, W. E. 1996, AJ, 112, 1487

Horne, K. 1986, PASP, 98, 609

Hoyle, F., \& Schwarzschild, M. 1955, ApJS, 2, 1

Kurucz, R. L. 1992, in The Stellar Populations of Galaxies, ed. B. Barbuy, \& A. Renzini, IAU Symp., 149, 225

Kurucz, R. 1993, ATLAS9 Stellar Atmosphere Programs and $2 \mathrm{~km} \mathrm{~s}^{-1}$ grid, Kurucz CD-ROM No. 13, Cambridge, Mass.: Smithsonian Astrophysical Observatory, 13
Michaud, G. 1970, ApJ, 160, 641

Michaud, G., Vauclair, G., \& Vauclair, S. 1983, ApJ, 267, 256

Moehler, S. 2001, PASP, 113, 1162

Moehler, S., Heber, U., \& de Boer, K. S. 1995, A\&A, 294, 65

Moehler, S., Heber, U., \& Rupprecht, G. 1997, A\&A, 319, 109

Moehler, S., Sweigart, A. V., \& Catelan, M. 1999, A\&A, 351, 519

Moehler, S., Sweigart, A. V., Landsman, W. B., \& Heber, U. 2000, A\&A, 360, 120

Moehler, S., Landsman, W. B., Sweigart, A. V., \& Grundahl, F. 2003, A\&A, 405, 135

Moehler, S., \& Sweigart, A. V. 2006, A\&A, 455, 943

Momany, Y., Piotto, G., Recio-Blanco, A., et al. 2002, ApJ, 576, L65

Momany, Y., Cassisi, S., Piotto, G., et al. 2003, A\&A, 407, 303

Momany, Y., Bedin, L. R., Cassisi, S., et al. 2004, A\&A, 420, 605

Moni Bidin, C., Moehler, S., Piotto, G., et al. 2006a [arXiv: astro-ph/0606035]

Moni Bidin, C., Moehler, S., Piotto, G., et al. 2006b, A\&A, 451, 499

Napiwotzki, R., Green, P. J., \& Saffer, R. A. 1999, ApJ, 517, 399

Pace, G., Recio-Blanco, A., Piotto, G., \& Momany, Y. 2006, A\&A, 452, 493

Peacock, J. A. 1983, MNRAS, 202, 615

Peterson, R. C. 1983, ApJ, 275, 737

Peterson, R. C. 1985a, ApJ, 289, 320

Peterson, R. C. 1985b, ApJ, 294, L35

Piotto, G., Zoccali, M., King, I. R., et al. 1999, AJ, 118, 1727

Przybilla, N., Nieva, M. F., \& Edelmann, H. 2006, Baltic Astron., 15, 107

Ramırez, I., \& Meléndez, J. 2005, ApJ, 626, 465

Recio-Blanco, A., Piotto, G., Aparicio, A., \& Renzini, A. 2004, A\&A, 417, 597

Reid, I. N. 1997, AJ, 114, 161

Reid, N. 1998, AJ, 115, 204

Renzini, A., Bragaglia, A., Ferraro, F. R., et al. 1996, ApJ, 465, L23

Saffer, R. A., Bergeron, P., Koester, D., \& Liebert, J. 1994, ApJ, 432, 351

Sandage, A., \& Wallerstein, G. 1960, ApJ, 131, 598

Sandage, A., \& Wildey, R. 1967, ApJ, 150, 469

Sekiguchi, M., \& Fukugita, M. 2000, AJ, 120, 1072

Sosin, C., Dorman, B., Djorgovski, S. G., et al. 1997, ApJ, 480, L35

Sweigart, A. V. \& Catelan, M. 1998, ApJ, 501, L63

Tüg, H. 1977, The Messenger, 11, 7

van den Bergh, S. 1967, AJ, 72, 70

Vink, J. S., \& Cassisi, S. 2002, A\&A, 392, 553 